\title{
1 Lignin-containing photoactive resins for 3D printing by
}

\section{2 stereolithography}

3 Jordan T. Sutton ${ }^{1,2}$, Kalavathy Rajan ${ }^{1,3}$, David P. Harper*1, Stephen C. Chmely ${ }^{\star 1}$

4

5 1. Center for Renewable Carbon, The University of Tennessee Institute of Agriculture,

$6 \quad$ Knoxville, Tennessee 37996, United States

7 2. Department of Materials Science and Engineering, The University of Tennessee

8 Knoxville, Knoxville, Tennessee 37996, United States

9 3. Department of Biosystems Engineering and Soil Science, The University of

10 Tennessee Institute of Agriculture, Knoxville, Tennessee 37996, United States

12 Corresponding authors' email: dharper4@utk.edu, schmely@utk.edu

14 Keywords: lignin, 3D printing, stereolithography, additive manufacturing, resin 


\section{Abstract}

Generating compatible and competitive materials that are environmentally sustainable and economically viable is paramount for the success of additive manufacturing using renewable materials. We report the successful application of renewable, modified lignin-containing photopolymer resins in a commercial stereolithography system. Resins were fabricated within operable ranges for viscosity and cure properties, using up to $15 \%$ modified lignin by weight with the potential for higher amounts. A four-fold increase in ductility in cured parts with higher lignin concentration is noted as compared to commercial SLA resins. Excellent print quality was seen in modified lignin resins, with good layer fusion, high surface definition, and visual clarity. These materials can be used to generate new products for additive manufacturing applications and help fill vacant material property spaces, where ductility, sustainability, and application costs are critical. 


\section{Introduction}

Additive manufacturing offers greater freedom of design, quicker product development, and at a cheaper cost over traditional manufacturing techniques. In 2018, the global additive manufacturing (AM) market has a predicted value of $\$ 12.8$ billion. $^{1}$

The complexity offered by greater design freedom means that parts can be topologically optimized to improve mechanical properties and hybrid materials can be designed that can achieve material properties unobtainable with conventional material processing. ${ }^{2-3}$ However, one major hurdle for all AM technologies is in the design of new materials that are compatible with these AM processes but still comparable in cost and properties to those of traditional manufacturing. Renewable-sourced biomaterials are sustainable and abundant, giving them a competitive advantage over materials derived from fossil fuels. Hence, engineering novel biomaterials provides the opportunity to create cost-effective materials that reduce the environmental impact of additive manufacturing while meeting traditional material requirements.

Stereolithography (SLA) is an AM process that uses a bath of liquid photopolymer resin, a movable platform, and a laser UV source to cure resin layer by layer to build part geometries. The main limitation of SLA technology is the photopolymer build material. In the next decade, the market for 3D printing materials is expected to grow to at least $\$ 16$ billion, and a significant portion of that is predicted to be shared by photopolymers. ${ }^{1}$ Currently, these materials are few, limited in features, and expensive. ${ }^{4}$

Resins designed for AM processes are complex mixtures generally composed of a photoinitiator, monomers and oligomers, and other additives incorporated to achieve 
specific properties. Photosensitizers and UV blockers, for instance, can be added to shift cure wavelength and affect reaction kinetics. ${ }^{5}$ Resin formulations are finely tuned to work efficiently with the printer configuration and achieve the desired material properties. As expected, these complex formulations are costly to design and produce. In addition to cost, photopolymers typically exhibit issues with shrinkage, brittleness, and slow cure speed. In SLA, these problems are related to the photopolymerization reaction. Therefore, one approach to solving these problems is to control the reaction by altering the resin formulation.

Lignin is the world's second-most abundant natural polymer, and the only highvolume renewable feedstock composed of aromatics. ${ }^{6}$ Most of the lignin available is produced as a byproduct of cellulose production, and although much of it is used as a fuel source for these processes, some methods can generate up to $60 \%$ more lignin than is used during combustion. ${ }^{7}$ In fact, by 2022,62 million tons of lignin will be produced annually. ${ }^{8}$ Second-generation biofuels derived from lignocellulosic sources are poised to play an important role in alternative energy solutions, and the economic success of these biorefineries lies with co-product revenue streams.

Much work has gone toward the valorization of lignin for use in fuels, chemicals, and polymers. For instance, lignin could be used to replace costly polyacrylonitrile as a precursor material for producing carbon fibers (CF), and currently many groups are working towards improving the performance of lignin-derived versus traditionally-made CFs. ${ }^{9-11}$ Lignin also shows great promise for use in other sustainable soft materials, ${ }^{12}$ and several commercially viable products containing lignin have been investigated. ${ }^{13}$ 
In contrast to simply blending technical lignin with existing petrochemical-based polymers, chemical modifications to isolated technical lignin typically lead to improved chemical, physical, and thermal properties of the resulting product. In fact, these can even offer additional functionality, such as an increase in photoactivity. For instance, bio-plastics developed from acylated model-lignin compounds like syringol, creosol, and vanillin were shown to possess enhanced thermal stability and tunable viscoelastic properties. ${ }^{14-15}$ Bio-based epoxy resins made from depolymerized and glycidylated softwood lignin displayed superior flexural modulus and flexural strength. ${ }^{16}$ Our group has also recently demonstrated that engineered hydrogels containing methacrylatemodified hardwood lignin exhibited increase in water retention capacity and tunable mechanical properties. ${ }^{17}$ Moreover, alkene-functionalized lignin was successfully harnessed for photo-induced polymerization reactions. ${ }^{18}$ In short, these studies demonstrate that designing lignin building blocks via chemical modification of the $\mathrm{OH}$ groups is a feasible approach for formulating 3D printing resins with tunable properties.

We are interested in developing transformations of lignin to make it amenable to incorporation into new high-performance engineered materials. Here, we report on our efforts to produce photoactive resins that contain organosolv lignin isolated from hybrid poplar. We formulated these resins by chemically modifying the lignin macromolecules to contain methacrylate moieties. Then, we blended the lignin with commercially available resin components to formulate a complete resin. We used a commercial desktop 3D printer from Formlabs to print with these resins, and report on the spectroscopic, mechanical, and thermal properties of these materials. This work 
represents a platform from which the design of advanced photoactive resins for additive manufacturing purposes can commence.

\section{Experimental Section}

2.1. Reagents and materials. Lignin was isolated from pulp-grade wood chips of hybrid poplar (Populus trichocarpa $\times P$. deltoides) using an ethanol organosolv technique as described by Bozell and coworkers. ${ }^{19}$ Ethoxylated pentaerthritol tetraacrylate (SR494, Sartomer) and aliphatic urethane acrylate (Ebecryl 8210, Allnex) were used as resin bases. A monofunctional urethane acrylate (Genomer 1122, Rahn) was used as a reactive diluent. Diphenyl (2,4,6-trimethylbenzoyl) phosphine oxide (PLTPO, Esstech) and 2,2'-(2,5-thiophenediyl)bis(5-tert-butylbenzoxazole) (Benetex OB

Plus, Mayzo) were used as the resin photoinitiator and UV blocker, respectively.

Commercial PR48 resin was obtained from Colorado Photopolymer Solutions (Boulder, CO) for comparison purposes. 2-chloro-4,4,5,5-tetramethyl-1,3,2-dioxaphospholane (TMDP, Santa Cruz Biotechnology, Inc.) was stored in a vacuum desiccator to protect it from atmospheric moisture. Endo- $N$-hydroxy-5-norbornene-2,3-dicarboximide (SigmaAldrich) was used as received.

2.2. Lignin modification for resin formulation. The protocol for lignin acylation (Scheme 1) was adapted from the method for syringyl methacrylate synthesis as described by Epps and coworkers. ${ }^{14-15}$ Methacrylic anhydride (Alfa Aesar) was allowed to react with lignin (1.2 equiv of the lignin $\mathrm{OH}$ groups as measured by ${ }^{31} \mathrm{P} N M R$ ) in the presence of 4-dimethylaminopyridine (DMAP, Sigma-Aldrich) as a catalyst (0.04 equiv 
119 of methacrylic anhydride). The reaction mixture was incubated at $60^{\circ} \mathrm{C}$ for 48 hours and 120 the lignin thus acylated (lignin-M) was purified by quenching the byproducts and

121 unreacted substrate with a saturated solution of sodium bicarbonate. Lignin-M that 122 precipitated during the reaction was washed with copious amounts of water until the 123 washes reached neutral $\mathrm{pH}$. The resulting precipitate was dried under reduced pressure 124 at $40{ }^{\circ} \mathrm{C}$ for 48 hours and used for resin formulation.

125 Scheme 1. Synthesis scheme for lignin-M using methacrylic anhydride.

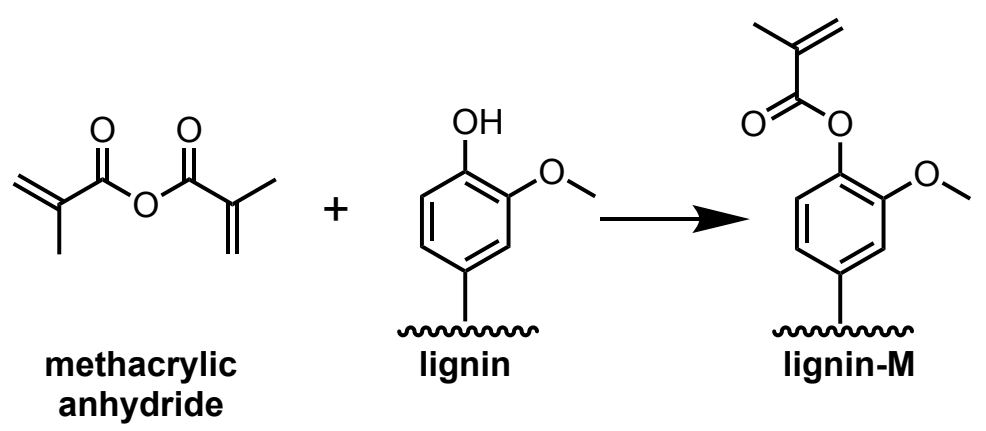

2.3. Lignin characterization. The hybrid poplar lignin and lignin-M were characterized using ${ }^{31} \mathrm{P}$ NMR and Fourier transform infrared (FTIR) spectroscopies to monitor the chemical changes in lignin and the efficiency of acylation reaction. Sample preparation and quantification of lignin $\mathrm{OH}$ groups was achieved using ${ }^{31} \mathrm{P}$ NMR spectroscopy as described by Balakshin and Capanema. ${ }^{20}$ Briefly, the lignin samples were phosphitylated using TMDP, and the resulting NMR spectra were phased and referenced to the chemical shift of the water-derived complex of TMDP ( $\delta 132.2 \mathrm{ppm})$. Quantification was achieved using endo- $N$-hydroxy-5-norbornene-2,3-dicarboximide as the internal standard. All ${ }^{31} \mathrm{P}$ NMR spectra were collected using a Varian 400-MR spectrometer (Varian Inc., Palo Alto, CA), operating at $161.92 \mathrm{MHz}$ and $25^{\circ} \mathrm{C}$. FTIR 
138 instrument (PerkinElmer, Llantrisant, UK), where the finely ground samples were

139 analyzed at 16 scans per spectrum and $4 \mathrm{~cm}^{-1}$ resolution. The FTIR peaks were

140 assigned based on previous reports, ${ }^{21-23}$ and the spectra were subjected to statistical

141 analysis using principal component analysis (PCA).

2.4. Resin formulation. The formulations of the $3-D$ printing resins used in this

143 investigation are presented in Table 1. The photoinitiator and UV blocker were weighed

144 and combined in a polypropylene container. The resin bases were added by weight in

145 increasing viscosity as follows: the monofunctional urethane acrylate (Genomer 1122),

146 the tetra-acrylate oligomer (SR494), and finally the aliphatic urethane acrylate (Ebecryl

147 8210). The partially formulated resin was mixed in a kinetic mixer (FlackTek,

148 Speedmixer Dac 150 FVZ) at 2400 RPM for 0.5 min. Lignin-M was then weighed and

149 added to the container and the resin was mixed again at 2400 RPM for $1 \mathrm{~min}$. The lignin 150 resins (LR) thus formulated (5-15\% lignin by weight, LR5, LR10, and LR15) were stored

151 in tightly sealed opaque containers to protect them from light.

152 Table 1. Composition of Modified Resins

\begin{tabular}{lllllll}
\hline Resin & $\begin{array}{l}\text { SR494 } \\
(w t \%)\end{array}$ & $\begin{array}{l}\text { Ebecryl } \\
8210(w t \%)\end{array}$ & $\begin{array}{l}\text { Genomer } \\
1122(w t \%)\end{array}$ & $\begin{array}{l}\text { Lignin-M } \\
(w t \%)\end{array}$ & $\begin{array}{l}\text { PL- } \\
\text { TPO } \\
(w t \%)\end{array}$ & $\begin{array}{l}\text { Mayzo } \\
\text { OB+ } \\
(w t \%)\end{array}$ \\
\hline PR48 & 39.78 & 39.78 & 19.89 & 0 & 0.4 & 0.16 \\
LR5 & 37.84 & 37.84 & 18.92 & 5 & 0.4 & 0 \\
LR10 & 35.84 & 35.84 & 17.92 & 10 & 0.4 & 0 \\
LR15 & 33.84 & 33.84 & 16.92 & 15 & 0.4 & 0 \\
\hline
\end{tabular}


2.5. Resin working curves. All resins were characterized for critical cure dosage

155

156

157

158

159

160

161

162

163

164

165

166

167

168

169

170

171

172

173

174

and penetration depth parameters using the working curve method outlined

elsewhere. ${ }^{24}$ This method relies on a relationship between cure thickness and dosage derived from the Beer-Lambert Law as shown below:

$$
C_{d}=D_{p} \cdot \ln (E)-D_{p} \cdot \ln \left(E_{c r i t}\right)
$$

where $C_{d}$ is the cure depth, $D_{p}$ is the penetration depth, $E_{\text {crit }}$ is the critical dosage exposure required to cure the resin, and $E$ is the dosage applied per layer. This dosage can be controlled by the laser scan velocity in accordance with the following relation:

$$
E=\frac{P_{L}}{V_{s} h_{s}}
$$

where $V_{s}$ is the scan line velocity, $h_{s}$ is the scan line spacing, and $P_{L}$ is the laser power.

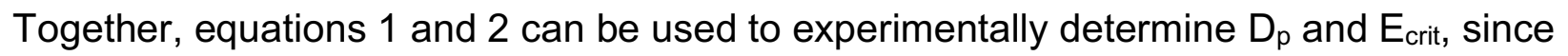
a plot of cure depth (thickness) versus the natural log of dosage (E) can be fit with a line whose slope is penetration depth $\left(D_{p}\right)$ and whose y-intercept (b) is given as

$$
b=-D_{p} \cdot \ln \left(E_{c r i t}\right)
$$

A rectangular model was designed using AutoCAD (Autodesk, Inc., San Rafael, CA) having dimensions $40 \times 64 \mathrm{~mm}^{2}$. It was composed of 32 squares that had varying thickness ranging from 5 to 20 layers. The model was printed directly on the resin bath to ensure that the resultant thicknesses were due only to the applied dosage. The 3D printed specimen was removed from the bath and cleaned of excess resin. The material thickness was measured for each square using a digital Vernier caliper and plotted against the natural log of the dosage. A linear regression was performed on the data to 
175 obtain the slope (corresponding to $D_{p}$ ) and y-intercept (related to the critical dosage as 176 described by equation 3 above).

177

178

179

180

181

182

183

184

185

186

187

188

189

190

191

192

193

194

195

196

197

2.6. 3D printing using lignin resins by stereolithography. Models for $3 \mathrm{D}$

printing were designed in AutoCAD, and all samples were generated using a Formlabs

(Formlabs, Inc., Somerville, MA) Form 1+ desktop stereolithography (SLA) printer, installed with a modified version of the printer's software called OpenFL. The SLA printer used a $120 \mathrm{~mW}$ Class-1 laser emitting at $405 \mathrm{~nm}$, and had a build volume of 125 $\times 125 \times 165 \mathrm{~mm}^{3}$. The 3D printing resins were stored and used at room temperature.

Custom print files for each resin were designed using dose calculations obtained from the resin working curves. The laser settings for printing the LR resins were obtained by modifying the laser-scan velocity that delivered the necessary dosage, as per eqn. (2).

Print files were designed to cure the resins of $50 \mu \mathrm{m}$ thickness. OpenFL was used to generate the scaffolds before printing. Tensile bars were built at a $45^{\circ}$ angle with scaffolds edited to avoid placement along the gauge length.

The printed parts were separated from the build platform and soaked in two consecutive baths of isopropanol for 10 min each to remove any uncured resin and scaffolds. A 400 W metal halide UV (arc) lamp (Uvitron International Inc., West Springfield, MA), with irradiance capacity of $200 \mathrm{~mW} / \mathrm{cm}^{2}$ at a height of $7.62 \mathrm{~cm}$ was used to post-cure the printed parts for $3 \mathrm{~min}$.

2.7. Materials characterization. The viscosity of the formulated resins was measured using a TA Instruments AR-G2 rheometer with cone and plate geometry. The rheometer was configured with a $40 \mathrm{~mm}$ diameter cone at a truncation gap of $56 \mu \mathrm{m}$. Tests were performed over a range of shear rates from 0.1 to $100 \mathrm{~Hz}$ at $25^{\circ} \mathrm{C}$. 

spectroscopy, where the solids samples were directly analyzed on the crystal of an ATR accessory attachment. Five individual spectra were collected for all samples.

201

202

203

204

205

206

207

208

209

210

211

212

213

214

215

216

217

218

219

Thermogravimetric analysis (TGA) was performed using a PerkinElmer Pyris 1 TGA.

Each test was programmed to run from $30{ }^{\circ} \mathrm{C}$ to $900^{\circ} \mathrm{C}$ at a heating rate of $20^{\circ} \mathrm{C} / \mathrm{min}$.

All tests were done under a continuous nitrogen flow of $10 \mathrm{~mL} / \mathrm{min}$.

Ultimate tensile strength and Young's modulus of the cured samples were obtained using an Instron 5567 dual column universal testing machine. A $30 \mathrm{kN}$ static load cell was used. Tests were performed following the ASTM D638 standard according to type $\mathrm{V}$ specimen dimensions. A $1 \mathrm{~mm} / \mathrm{min}$ extension rate was applied for all tests. Scaffold marks on printed samples were removed by sanding prior to testing to avoid any stress concentration.

Scanning electron microscopy (SEM) was performed using a Phenom ProX with an accelerating voltage of $10 \mathrm{kV}$. Samples were cut to size and cleaned of debris prior to mounting.

\section{Results and Discussion}

3.1. Modified lignin characterization. Lignin-M, obtained via acylation of $\mathrm{OH}$ groups of hybrid poplar lignin with methacrylic anhydride (Scheme 1), was characterized using ${ }^{31} \mathrm{P}$ NMR and FTIR spectroscopy. Figure 1a depicts the FTIR spectra, in which lignin-M had a significant reduction in $\mathrm{OH}$ functional groups $\left(3403 \mathrm{~cm}^{-1}\right)$. This is expected, as the number of $\mathrm{OH}$ groups is depleted as the acylation reaction takes place (Scheme 1). The FTIR spectra indicate an increase in methacrylate functional groups 
220 attached to the modified lignin $\mathrm{OH}$ groups, as evidenced by increases in peaks

221 corresponding to $\mathrm{C}=\mathrm{O}$ stretches, $\mathrm{C}-\mathrm{O}-\mathrm{C}$ stretches, and in plane $-\mathrm{CH}_{2}$ bending vibrations $222\left(1723,1131\right.$ and $943 \mathrm{~cm}^{-1}$, respectively).

${ }^{31} \mathrm{P}$ NMR spectroscopy is another method used to quantify the changes in lignin

$224 \mathrm{OH}$ groups, and is particularly useful to detect differences among substitution at the

225 aliphatic, syringyl (S), guaiacyl $(\mathrm{G})$, para-hydroxy $(\mathrm{H})$, and carboxylic acid $(\mathrm{COOH})$

226 hydroxyl groups. As shown in Figure 1b, OH groups in lignin-M undergo substantial

227 substitution, where $92 \%$ of these are acylated. The scant amounts of $\mathrm{COOH}$ groups $(0.1$

$228 \mathrm{mmol} / \mathrm{g}$ ) observed in lignin-M might have originated from residual methacrylic acid,

229 which is a byproduct of lignin modification.
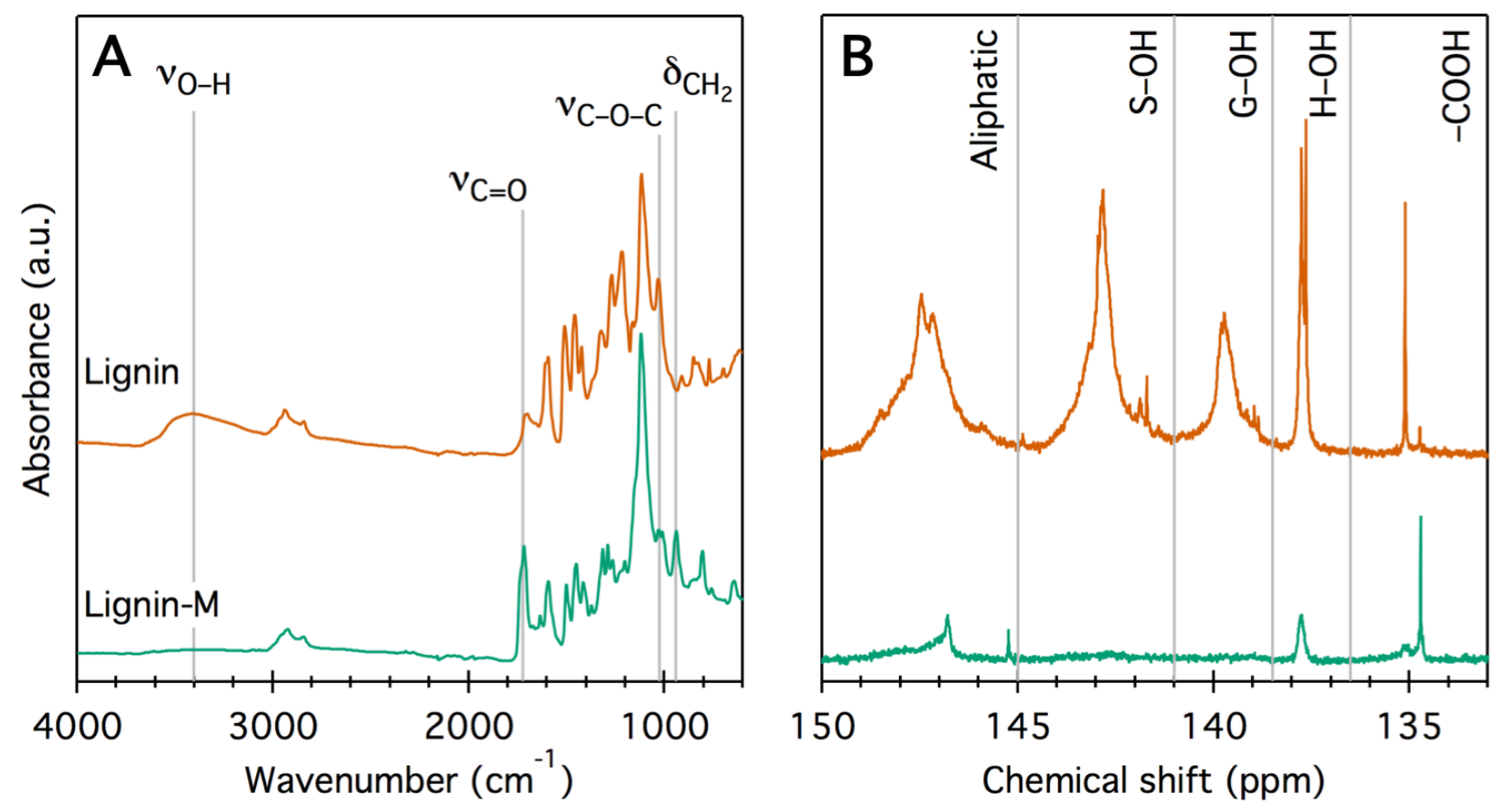

231 Figure 1. (A) Fourier transform infrared spectra of hybrid poplar lignin before (top,

232 brown) and after (bottom, green) methacrylate modification of the $\mathrm{OH}$ groups to afford

233 lignin-M. Disappearance of the $\mathrm{V}_{\mathrm{O}-\mathrm{H}}$ peak indicates substitution at the hydroxyl groups, 
234

235

236

237

238

239

240

241

242

243

244

245

246

247

248

249

250

251

252

253

254

255

256

and an increase in $\mathrm{v}_{\mathrm{C}=\mathrm{O}}, \mathrm{v}_{\mathrm{C}-\mathrm{O}-\mathrm{C}}$, and $\delta_{\mathrm{CH} 2}$ indicate the presence of a methacrylate moiety. (B) ${ }^{31} \mathrm{P}$ NMR spectra of the same samples (color scheme and position identical) with integration ranges (taken from Balakshin and $\mathrm{Capanem}^{20}$ ) also indicate substitution at the hydroxyl groups, including the syringyl, guaiacyl, and p-hydroxy (S, $\mathrm{G}$, and $\mathrm{H}$, respectively) aromatic hydroxyl groups. The shifted $-\mathrm{COOH}$ peak in lignin-M is due to residual methacrylic acid, a byproduct of the methacrylation reaction.

3.2. LR formulations. We used lignin-M as a source of acrylate oligomers and reactive diluent at 5 to $15 \%$ by weight to formulate our 3D printing resins. To ensure that lignin-M was incorporated into the resin bases homogenously, we created the LR mixtures in small batches and allowed them to rest for $48 \mathrm{~h}$. Unmodified lignin dissolved poorly in acrylate-based resins, whereas lignin-M showed significant improvement in homogeneity owing to the compatibility of its methacrylate functional groups (Figure S1).

We identified resin viscosity as a critical parameter for compatibility with an additive printing mechanism because of its role in affecting the resin recoating procedure over the curing surfaces between two contiguous layers. Fast laser scan speeds meant that, in general, the speed at which the part can be printed is determined by how quickly the printer can reset for a new layer. Higher viscosities result in longer wait times for the recoating procedure and overall longer printing duration. The viscosity measurements for LR5-15 (Figure 2) showed that they displayed Newtonian behavior and had a viscosity range from 0.44 to $1.66 \mathrm{~Pa} \cdot \mathrm{s}$. Commercial manufacturers have reported viscosities in the ranges of 0.85 to $4.5 \mathrm{~Pa} \cdot \mathrm{s}$ for their 3D printing resins, so LR515 are evidently viable for creating equivalent print designs. The measured viscosity of 
257 LR5-15 increased with increasing lignin-M content, which implies a limit to the amount 258 of lignin the base resin can host and still be within a usable viscosity range without 259 additional diluent.

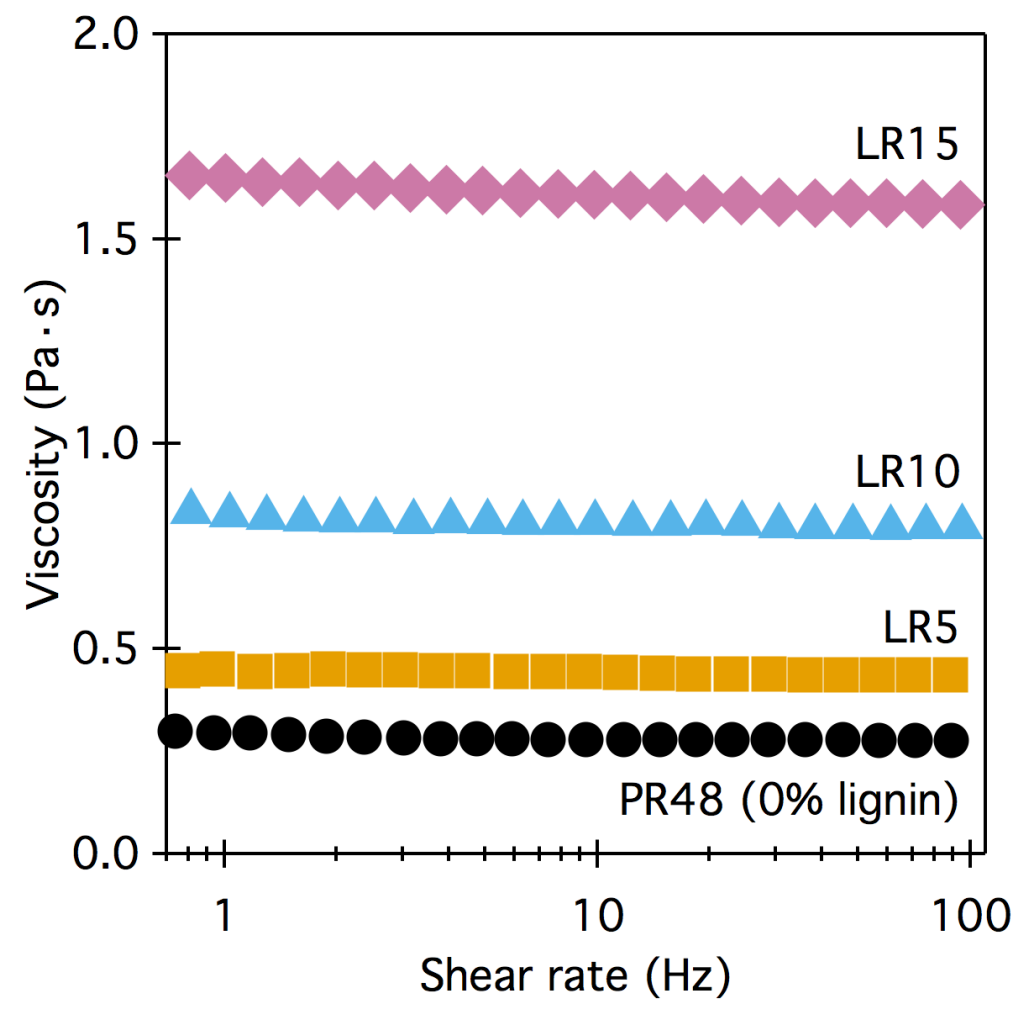

261 Figure 2. Plot of viscosity versus shear rate of photo curable acrylate resins containing 262 various amounts of lignin-M. Viscosity increases with increasing amounts of lignin as 263 compared to the commercial PR48 resin. 3.3. Resin cure properties. The working curve approach is a basic model for 265 formulating SLA resins, since it provides a measurement of threshold energy required to 266 initiate photopolymerization ( $\left.E_{\text {crit }}\right)$ and also helps to evaluate the resin cure properties. ${ }^{24}$ 267 The working curves generated for all LR formulations are shown in Figure 3, and the 268 corresponding cure properties are listed in Table 2, along with the target dosage for a 
269 printing a layer with a thickness of $50 \mu \mathrm{m}$ (a photograph of the windowpane rectangle 270 used to generate working curves is provided as Figure S2).

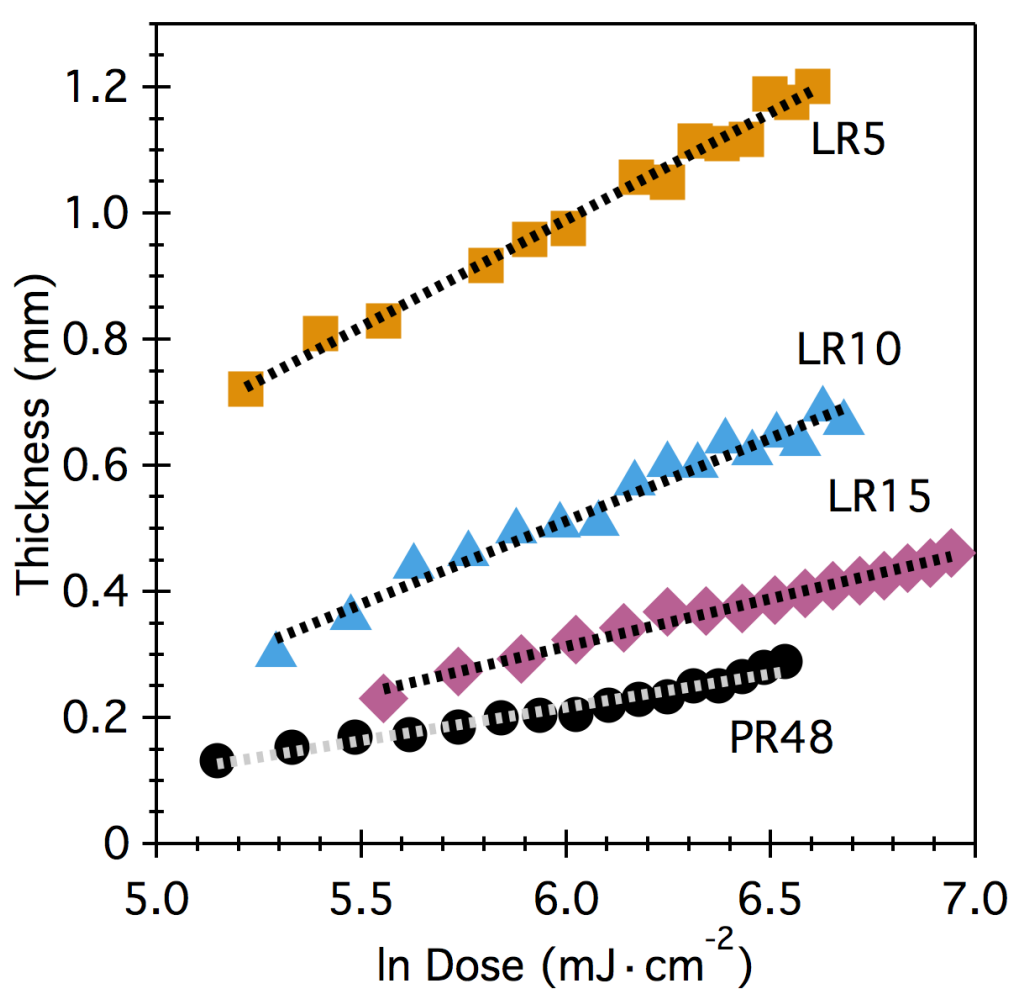

272 Figure 3. Working curves showing cure thickness as a function of the natural log of UV

273 dosage for PR48 and LR formulations. Linear regressions are shown as dotted lines, 274 and the related values are tabulated in Table 2 from Equations 1-3 above.

275 Penetration depth $\left(D_{p}\right)$ is the distance photons travel in the resin before they are 276 absorbed by a resin component. Resins with high $D_{p}$ will allow more photons to pass 277 farther into the resin, causing it to potentially cure thicker than a resin with a lower $D_{p}$. 278 This results in poor resolution control and nonuniformity in the build geometry. Typically, 279 SLA resins contain a UV blocker (Mayzo OB+ in PR48, see Table 1) to tune the $D_{p}$ and 280 reduce the required precision during printing. 

bonds), we expected that it would readily absorb UV photons and potentially serve the same role as the UV blocker. Consequently, we prepared all LR formulations without Mayzo OB+. As is evident from the data in Table 2, even up to $15 \mathrm{wt}$ \% lignin-M, Dp for the LR formulations was still higher than that for PR48, which contains 0.16 wt- $\%$ UV blocker. Surprisingly, the added lignin does not appear to retard the penetration of the UV photons required to initiate the polymerization reactions. As expected, $D_{p}$ decreased 288 in resins with higher amounts of incorporated lignin-M. The photoactivity of lignin results 289 in light scattering and absorption, which reduces the transmission through the resin.

290 Evidently, lignin-M in the LR resins could act not only as a structural feature but also as 291 a UV blocker, owing to its ability to scatter and absorb photons. We are currently 292 investigating the efficacy of lignin to be used as a specialized UV blocker in these 293 formulations.

294 Table 2. Cure parameters for tested resin formulations

\begin{tabular}{lll}
\hline $\begin{array}{l}\text { Resin } \\
\text { Formulation }\end{array}$ & $\mathrm{D}_{p}(\mathrm{~mm})$ & $\mathrm{E}_{\text {crit }}\left(\mathrm{J} \cdot \mathrm{cm}^{-2}\right)$ \\
\hline PR48 & $0.105(5)$ & $0.05(2)$ \\
LR5 & $0.34(1)$ & $0.022(5)$ \\
LR10 & $0.26(1)$ & $0.06(2)$ \\
LR15 & $0.152(5)$ & $0.05(1)$
\end{tabular}

295 Relative uncertainties in the final digit are reported in parentheses.

$296 \quad D_{p}$ : penetration depth; $E_{\text {crit: }}$ Critical cure dosage 
Interestingly, the critical cure dosage $\left(\mathrm{E}_{\text {crit }}\right)$ was lower for the lignin-M containing

299

300

301

302

303

304

305

306

307

308

309

310

311

312

resins, even at $15-w t \%$ loading. Although a higher critical cure dosage will result in a larger starting dosage and could significantly increase the curing time, evidently the presence of lignin does not deter resin curing. However, since the LR formulations do not include a UV blocker, the presence of which would increase the critical cure dosage, we are unable to unambiguously determine the reason for the change in $E_{\text {crit. }}$ There is certainly a complex interplay between penetration depth and critical cure dosage that depends upon the amount of lignin in each sample, since lignin is known to be excited by UV light and quench the free radicals by undergoing redox reactions resulting in the formation of quinones. ${ }^{25} \mathrm{We}$ are currently investigating these phenomena further.

\subsection{Characterization of 3D printed and cured resins. We used FTIR} spectroscopy of the polymerized resins and analysis of FTIR spectra (Figure S3) by PCA to probe the chemical structure of the cured resins. From these, we determined that the lignin-M containing polymers were significantly different than the control (Figure 4a).

As indicated by the PC1 loadings plot (Figure 4b), the largest difference between PR48 and the LR formulations is due to the presence of unconjugated carbonyl bonds $(C=O)$, acrylate ester bonds $(C-O-C)$ and in-plane bending of $C=C$ groups $(1723,1191$, and $1407 \mathrm{~cm}^{-1}$, respectively) in PR48. These can be attributed to unreacted acrylate groups in PR48 due to an incomplete cure. We further observed these results from scanning electron micrographs (vide infra) which we attribute to sub-optimal printing parameters (see Figure 6 below). This suggests that the LR formulations were more fully cured than the commercial PR48 formulation using the printing parameters we 
321 selected. Additionally, the PC2 loadings plot (Figure 4c) indicates that LR15 displayed

322 higher asymmetric and symmetric stretching vibrations of $\mathrm{C}-\mathrm{H}$ bonds (at 2923 and 2855

$323 \mathrm{~cm}^{-1}$, respectively). This could possibly be attributed to the additional aliphatic groups in

324 lignin, although this difference between the resins is relatively minor as reflected by the

325 low percentage of contribution to the data variance (15\%).
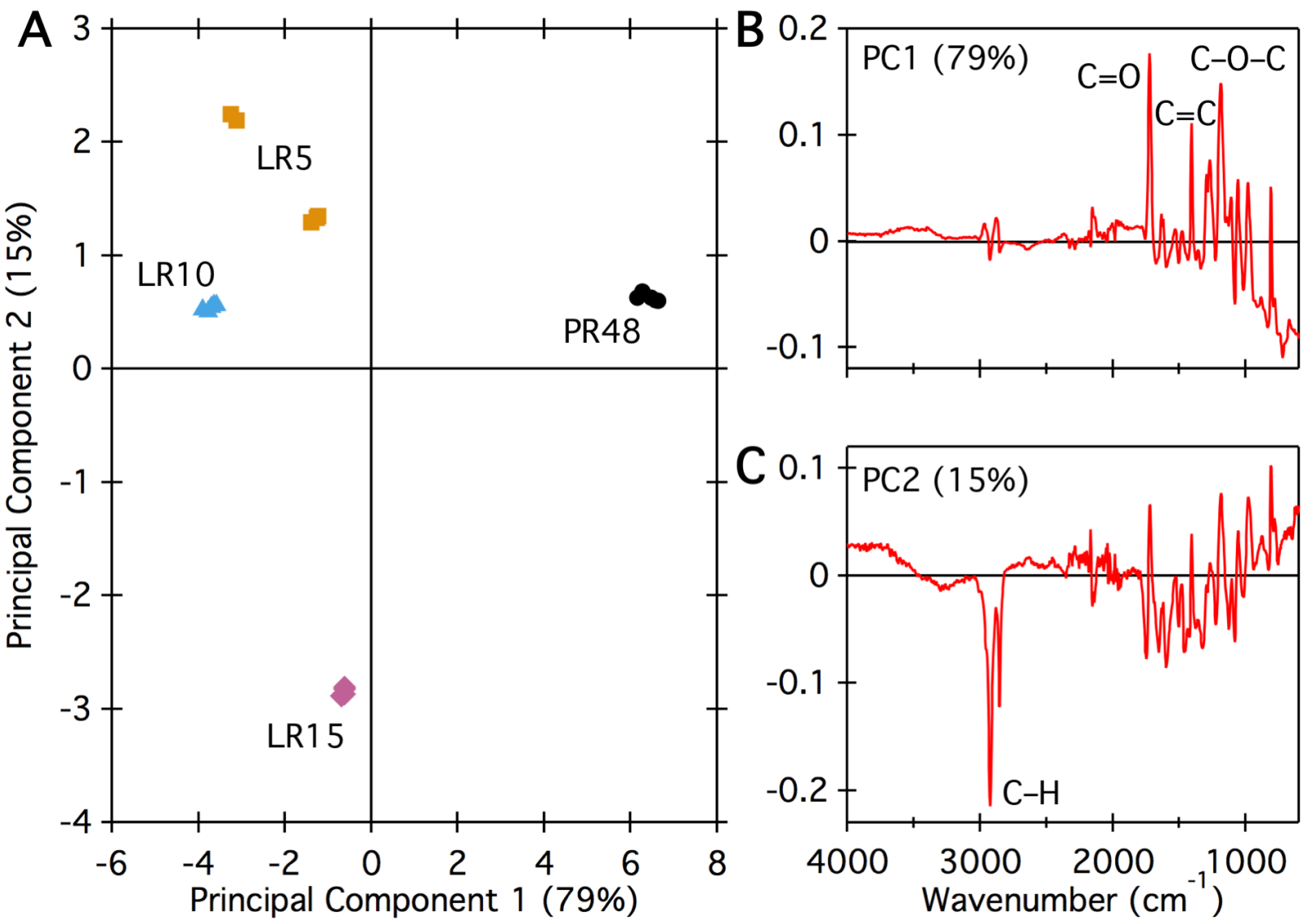

327 Figure 4. (A) Scores plot of the principal component analysis (PCA) of the FTIR spectra 328 of printed and cured PR48, LR5, LR10, and LR15 resin formulations. The labeling 329 scheme is identical to Figure 2. (B) Loadings plot of principal component 1 (PC1), which 330 accounts for $79 \%$ of the data variance. Stretching frequencies for $\mathrm{C}=\mathrm{O}, \mathrm{C}=\mathrm{C}$, and $\mathrm{C}-\mathrm{O}$ 
$331 \mathrm{C}$ moieties are noted. (C) Loadings plot of PC2, which accounts for $15 \%$ of the data 332 variance. Stretching frequencies for $\mathrm{C}-\mathrm{H}$ moieties are noted. We performed tensile tests on the cured resins, and the results of these experiments are summarized in Table 3. Interestingly, the cured resins displayed a 335 transition from brittle to ductile behavior in those that contain lignin-M. The elastic 336 modulus showed a $43 \%$ decrease from $0.65 \mathrm{GPa}$ with no lignin to $0.37 \mathrm{GPa}$ with $15 \%$ 337 lignin-M. Likewise, the ductility increased from a measured $1.87 \%$ elongation at break 338 for the control to $7.62 \%$ for LR15. This indicated that lignin-M was acting as a plasticizer 339 in the resin system. We attribute this phenomenon to the fact that the modified lignin 340 molecules were introducing side chains in the polyacrylate that reduced the chain-to341 chain interaction forces. ${ }^{26}$ Photopolymers are usually brittle, so such an improvement 342 could prove useful in increasing the toughness of parts printed by stereolithography.

343 Table 3. Mechanical testing of molded resins

\begin{tabular}{llll}
\hline Resin & $E(G P a)$ & $\sigma_{R}(\mathrm{MPa})$ & $\varepsilon_{R}(\%)$ \\
\hline PR48 & $0.65(5)$ & $11.0(4)$ & $1.87(8)$ \\
LR5 & $0.64(2)$ & $11(1)$ & $3.0(1)$ \\
LR10 & $0.48(2)$ & $18(3)$ & $6(1)$ \\
LR15 & $0.37(2)$ & $15(8)$ & $7.6(1)$
\end{tabular}
the cured LR resins exhibited different degradation onset temperatures when compared 
349 to the control. The mechanism of thermal decomposition of cured polyacrylate resins

350 such as PR48 and the related LR formulations has been the focus of intense

351 investigation by several groups. The rate of thermal decomposition of PR48 accelerates

352 as the temperature increases as evident by the first derivative of the TG curve (Figure 5,

353 inset). This phenomenon is known to be due to a complex combination of kinetically

354 regulated chain scission and depolymerization reactions. ${ }^{27}$ In case of the cured LR

355 formulations, we attribute the small weight loss at about $185^{\circ} \mathrm{C}$ to scission of the ether

356 bonds that link lignin-M to the growing polyacrylate polymer and the related

357 decomposition of the lignin-M oligomers, owing to the enhanced lability of $\mathrm{C}-\mathrm{O}$ bonds in 358 comparison to $\mathrm{C}-\mathrm{C}$ bonds. ${ }^{28}$

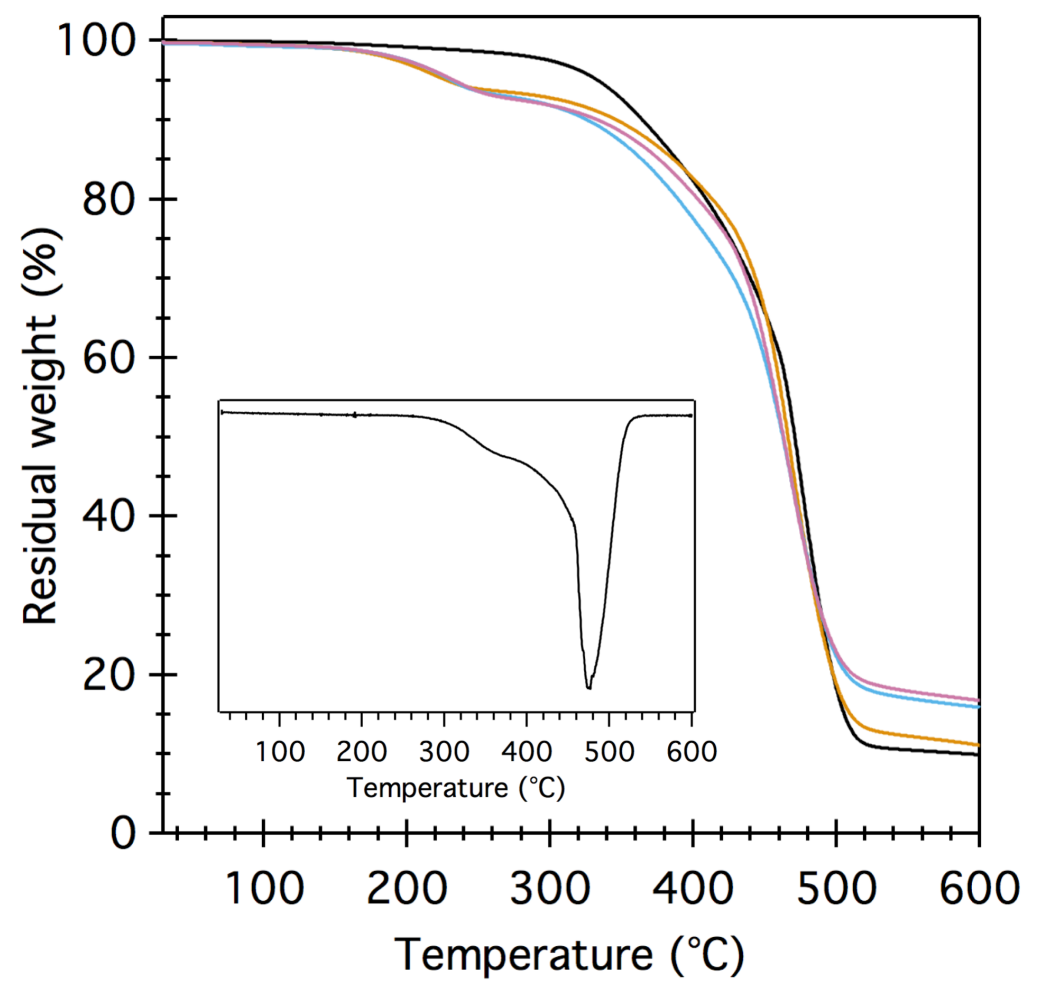

360 Figure 5. TGA curves (color scheme identical to Figure 2) for 3D printed commercial 361 PR48 resin and LR formulations. The derivative of the weight curve (inset) 
demonstrates the change in rate of thermal decomposition of PR48. The decomposition of the LR formulations at $\sim 185^{\circ} \mathrm{C}$ is evidence of thermolysis of the ether bonds that link lignin-M to the poly(acrylate) backbone.

3.5. Morphology and print quality. We used scanning electron microscopy to characterize the 3D printed surfaces (Figure 6). The surface of cured PR48 showed significant gaps between layers, due to a lack of fusion between them. These defects were regularly aligned along the edges of the line scan width where the dosage will be at minimum, whereas the fusion at the center of the cross-line scans extended between the layers. Since the print did not fail on the build platform, we attribute this phenomenon to a higher required UV dosage to attain inter-layer adhesion in PR48.

Evidently, at the print settings we have chosen for this study, optimal addition of lignin-M could enhance the photo-reactivity of the acrylate resins, thereby producing better prints at lower UV dosages.

LR5 provided the best print quality with relatively smooth surfaces and excellent layer adhesion, despite printing with the fastest laser scan velocity and at the lowest dosage. Print surfaces for LR10 and LR15 had small regions of poor fusion along print lines. These voids tended to be grouped together, suggesting that the presence of lignin-M disrupted the assemblage of polyacrylates due to cross-linking with lignin. Excepting these small regions, the surfaces of the 3D printed LR formulations showed complete fusion between layers, which emphasizes not only that lignin-M is compatible with SLA technology at the print layer level, but also that lignin-M shows great potential as a binding agent for improved print quality. 

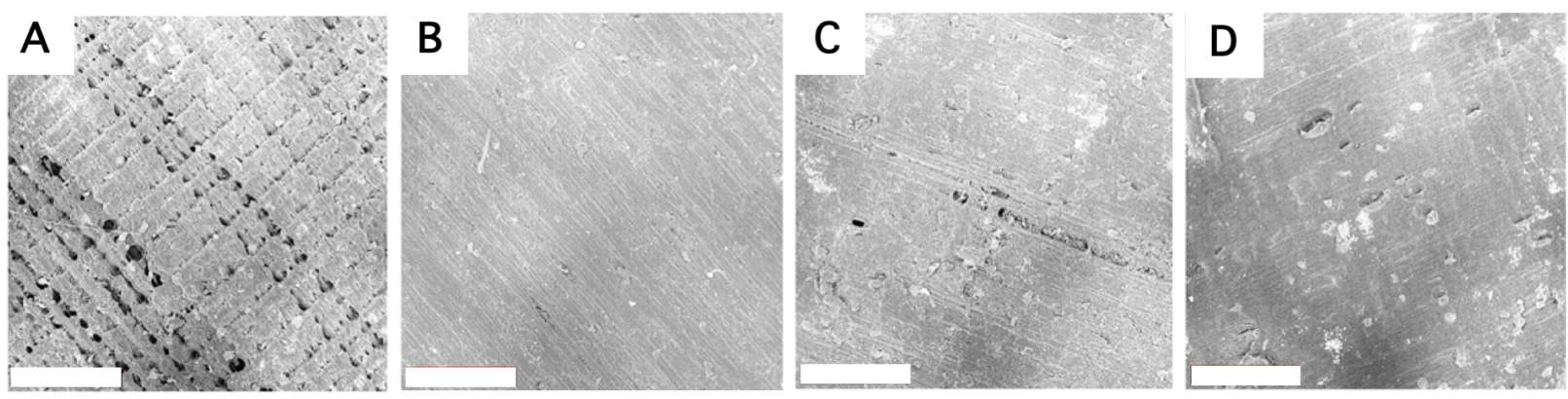

385

386

387

Figure 6. SEM images of the print surfaces for PR48 (A), LR5 (B), LR10 (C), and LR15 (D) showing an increase in surface roughness for LR formulations. Scale bars (white rectangles) denote $300 \mu \mathrm{m}$.

Visual evaluation of the print quality showed that the LR formulations displayed good build geometries compared to that of the commercial resin (Figure 7). High resolution and edge definition is achieved with the designed print settings. A significant degree of curling is present in the cured LR5 print because of the fast cure rate. Faster cure rates generally result in uneven shrinkage, causing a build-up of residual stresses and deformation of the material. Shrinkage can be reduced in this case either by modifying the resin formulation such that it has better cure properties or by changing the post-cure process. We did not observe this problem in the slower curing LR10 print.

Transparency was reduced with higher amounts of lignin-M, but the resins did not cure fully opaque. Thus, pigmentation could be applied to change the resin color, however, the brown coloration of lignin may limit the range of colors that could be used successfully. 


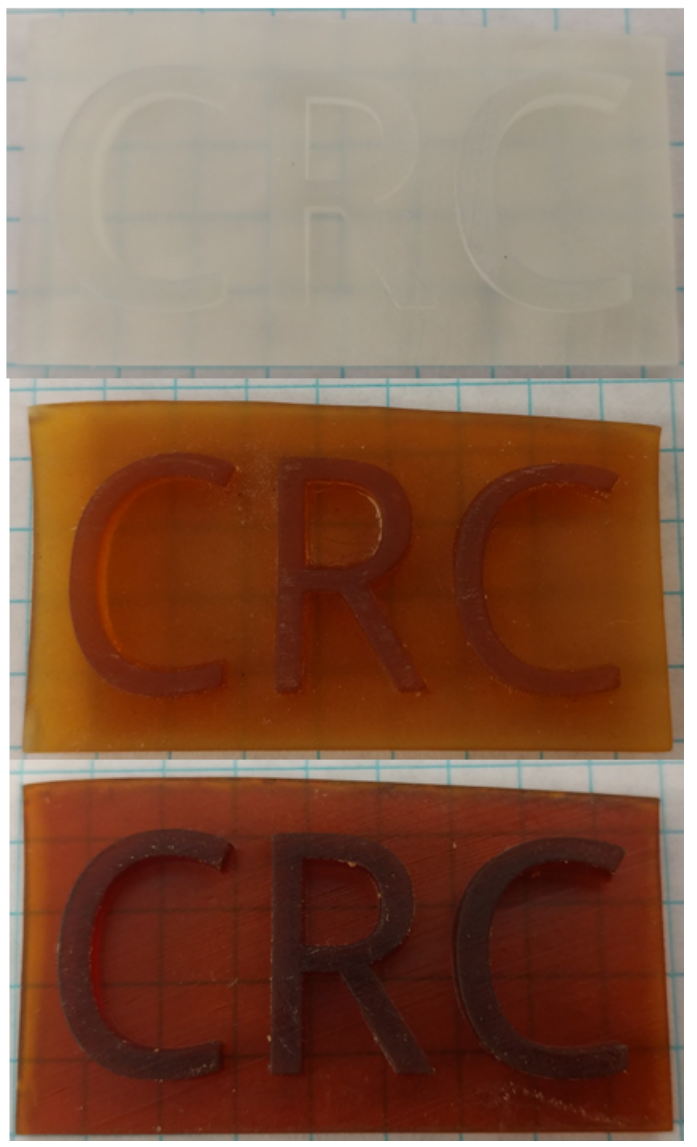

401 Figure 7. Photographs of 0\% (PR48, top), 5\% (LR5, middle), and 10\% (LR10, bottom)

402 lignin showing the effect on print quality. Even lignin-containing samples show

403 translucence, suggesting they are amenable to pigmentation.

\section{Conclusions}

In this work, we generated new photo-active acrylate resins by mixing

406 commercially available resin components with acylated organosolv lignin. The resulting

407 mixtures contained up to $15 \%$ by weight lignin and were used to produce 3D prints via

408 SLA technology. The lignin-containing resin formulations exhibited increased ductility

409 but decreased thermal stability when compared to the commercially available control

410 resin. Overall, we used the LR formulations to generate uniformly fused, high-resolution 
411 prints that display enhanced material toughness with a lower UV dosage. Future work

412 will focus on investigating the role that lignin plays as a UV blocker in these resin

413 formulations and using strategies to link functional monomers to lignin using $\mathrm{C}-\mathrm{C}$ bonds 414 instead of thermally labile $\mathrm{C}-\mathrm{O}$ bonds.

416 Acknowledgments

417 This project was supported by funds from the University of Tennessee AgResearch

418 Innovation Fund. Additional funds were provided by the Southeastern Sun Grant Center

419 and the US Department of Transportation, Research and Innovative Technology

420 Administration DTOS59-07-G-00050. D.P.H. and S.C.C. also acknowledge support from

421 the USDA National Institute of Food and Agriculture, Hatch Project 1012359. 


\section{References}

423

424

425

426

427

428

429

430

431

432

433

434

435

436

437

438

439

440

441

442

443

444

445

446

447

448

449

450

451

452

453

454

455

456

457

458

459

460

1. Wohlers, T. T.; Caffrey, T., Wohlers report $2015: 3 D$ printing and additive manufacturing state of the industry annual worldwide progress report. Wohlers Associates: Fort Collins, Colo., 2015; p 314 p.

2. Ashby, M., Hybrid Materials to Expand the Boundaries of Material-Property Space. J Am Ceram Soc 2011, 94, S3-S14. DOI: 10.1111/j.15512916.2011.04559.x

3. Babu, S. S.; Goodridge, R., Additive manufacturing. Mater Sci Tech-Lond 2015, 31 (8), 881-883. DOI: 10.1179/0267083615z.000000000929

4. Melchels, F. P. W.; Feijen, J.; Grijpma, D. W., A review on stereolithography and its applications in biomedical engineering. Biomaterials 2010, 31 (24), 61216130. DOI: 10.1016/j.biomaterials.2010.04.050

5. Gibson, I.; Rosen, D.; Stucker, B., Vat Photopolymerization Processes. In Additive Manufacturing Technologies : 3D Printing, Rapid Prototyping, and Direct Digital Manufacturing. $2^{\text {nd }}$ ed.; Springer: New York, NY, 2015; pp 63-106.

6. Tuck, C. O.; Perez, E.; Horvath, I. T.; Sheldon, R. A.; Poliakoff, M., Valorization of Biomass: Deriving More Value from Waste. Science 2012, 337 (6095), 695699. DOI: $10.1126 /$ science. 1218930

7. Sannigrahi, P.; Ragauskas, A. J., Characterization of Fermentation Residues from the Production of Bio-Ethanol from Lignocellulosic Feedstocks. J Biobased Mater Bio 2011, 5 (4), 514-519. DOI: 10.1166/jbmb.2011.1170

8. Ragauskas, A. J.; Beckham, G. T.; Biddy, M. J.; Chandra, R.; Chen, F.; Davis, M. F.; Davison, B. H.; Dixon, R. A.; Gilna, P.; Keller, M.; Langan, P.; Naskar, A. K.; Saddler, J. N.; Tschaplinski, T. J.; Tuskan, G. A.; Wyman, C. E., Lignin Valorization: Improving Lignin Processing in the Biorefinery. Science 2014, 344 (6185), 709-+. DOI: ARTN 124684310.1126/science.1246843

9. Hosseinaei, O.; Harper, D. P.; Bozell, J. J.; Rials, T. G., Role of Physicochemical Structure of Organosolv Hardwood and Herbaceous Lignins on Carbon Fiber Performance. ACS Sustainable Chemistry \& Engineering 2016, 4 (10), 57855798. DOI: 10.1021/acssuschemeng.6b01828 
489 490

491

492

493 494

10. Hosseinaei, O.; Harper, D. P.; Bozell, J. J.; Rials, T. G., Improving Processing and Performance of Pure Lignin Carbon Fibers through Hardwood and Herbaceous Lignin Blends. International Journal of Molecular Sciences 2017, 18 (7). DOI: 10.3390/ijms18071410

11. Mainka, H.; Tager, O.; Korner, E.; Hilfert, L.; Busse, S.; Edelmann, F. T.; Herrmann, A. S., Lignin - an alternative precursor for sustainable and costeffective automotive carbon fiber. J Mater Res Technol 2015, 4 (3), 283-296. DOI: 10.1016/j.jmrt.2015.03.004

12. Upton, B. M.; Kasko, A. M., Strategies for the Conversion of Lignin to High-Value Polymeric Materials: Review and Perspective. Chem Rev 2016, 116 (4), 22752306. DOI: $10.1021 /$ acs.chemrev.5b00345

13. Graichen, F. H. M.; Grigsby, W. J.; Hill, S. J.; Raymond, L. G.; Sanglard, M.; Smith, D. A.; Thorlby, G. J.; Torr, K. M.; Warnes, J. M., Yes, we can make money out of lignin and other bio-based resources. Industrial Crops and Products 2017, 106, 74-85. DOI: 10.1016/j.indcrop.2016.10.036

14. Holmberg, A. L.; Nguyen, N. A.; Karavolias, M. G.; Reno, K. H.; Wool, R. P.; Epps, T. H., Softwood Lignin-Based Methacrylate Polymers with Tunable Thermal and Viscoelastic Properties. Macromolecules 2016, 49 (4), 1286-1295. DOI: 10.1021/acs.macromol.5b02316

15. Holmberg, A. L.; Reno, K. H.; Nguyen, N. A.; Wool, R. P.; Epps, T. H., Syringyl Methacrylate, a Hardwood Lignin-Based Monomer for High-T-g Polymeric Materials. Acs Macro Lett 2016, 5 (5), 574-578. DOI: 10.1021/acsmacrolett.6b00270

16. van de Pas, D. J.; Torr, K. M., Biobased Epoxy Resins from Deconstructed Native Softwood Lignin. Biomacromolecules 2017, 18 (8), 2640-2648. DOI: 10.1021/acs.biomac.7b00767

17. Rajan, K.; Mann, J. K.; English, E.; Harper, D. P.; Carrier, D. J.; Rials, T. G.; Labbé, N.; Chmely, S. C., Sustainable Hydrogels Based on Lignin-Methacrylate Copolymers with Enhanced Water Retention and Tunable Material Properties. Biomacromolecules 2018, 19 (7), 2665-2672. DOI: 10.1021/acs.biomac.8b00282

18. Liu, H.; Chung, H., Visible-Light Induced Thiol-Ene Reaction on Natural Lignin. ACS Sustainable Chemistry \& Engineering 2017, 5 (10), 9160-9168. DOI: 10.1021/acssuschemeng.7b02065 
19. Bozell, J. J.; Black, S. K.; Myers, M.; Cahill, D.; Miller, W. P.; Park, S., Solvent fractionation of renewable woody feedstocks: Organosolv generation of biorefinery process streams for the production of biobased chemicals. Biomass Bioenerg 2011, 35 (10), 4197-4208. DOI: 10.1016/j.biombioe.2011.07.006

20. Balakshin, M.; Capanema, E., On the Quantification of Lignin Hydroxyl Groups with P-31 and C-13 Nmr Spectroscopy. J Wood Chem Technol 2015, 35 (3), 220-237. DOI: 10.1080/02773813.2014.928328

21. Schwanninger, M.; Rodrigues, J. C.; Pereira, H.; Hinterstoisser, B., Effects of short-time vibratory ball milling on the shape of FT-IR spectra of wood and cellulose. Vib Spectrosc 2004, 36 (1), 23-40. DOI: 10.1016/j.vibspec.2004.02.003

22. Lv, P.; Almeida, G.; Perré, P., TGA-FTIR Analysis of Torrefaction of Lignocellulosic Components (cellulose, xylan, lignin) in Isothermal Conditions over a Wide Range of Time Durations. Bioresources 2015, 10 (3). DOI: 10.15376/biores.10.3.4239-4251

23. Udagawa, A.; Sakurai, F.; Takahashi, T., In situ study of photopolymerization by fourier transform infrared spectroscopy. Journal of Applied Polymer Science 1991, 42 (7), 1861-1867. DOI: 10.1002/app.1991.070420707

24. Jacobs, P. F.; Reid, D. T., Rapid prototyping \& manufacturing : fundamentals of stereolithography. 1st ed.; Society of Manufacturing Engineers in cooperation with the Computer and Automated Systems Association of SME: Dearborn, MI, 1992; p 434 p.

25. Castellan, A.; Grelier, S.; Kessab, L.; Nourmamode, A.; Hannachi, Y., Photophysics and photochemistry of a lignin model molecule containing $\alpha-$ carbonyl guaiacyl and 4-hydroxy-3-methoxybenzyl alcohol moieties. J. Chem. Soc., Perkin Trans. 2 1996, (6), 1131-1138. DOI: 10.1039/p29960001131

26. Immergut, E. H.; Mark, H. F., Principles of Plasticization. In Plasticizationand Plasticizer Processes; Platzer, N. A. J., Eds.; American Chemical Society: Washington, DC, 1965; pp 1-26. DOI: 10.1021/ba-1965-0048.ch001

27. Holland, B. J.; Hay, J. N., The kinetics and mechanisms of the thermal degradation of poly(methyl methacrylate) studied by thermal analysis-Fourier transform infrared spectroscopy. Polymer 2001, 42 (11), 4825-4835. DOI: 10.1016/s0032-3861(00)00923-x 
$544 \quad$ 28. Kim, S.; Chmely, S. C.; Nimlos, M. R.; Bomble, Y. J.; Foust, T. D.; Paton, R. S.;

545 Beckham, G. T., Computational Study of Bond Dissociation Enthalpies for a

546

547 Large Range of Native and Modified Lignins. The Journal of Physical Chemistry Letters 2011, 2 (22), 2846-2852. DOI: 10.1021/jz201182w

548

549 